\title{
La importancia de percibir notoriedad e imagen de marca entre el personal de servicios de universidad
}

\author{
Javier Casanoves Boix ${ }^{1}$; Inés Küster Boluda ${ }^{2}$; Natalia Vila López ${ }^{3}$
}

\begin{abstract}
Resumen. A través de la presente investigación se pretende analizar el papel del capital de marca en el sector educativo entre el personal de administración y servicios. Para tal fin, se analizan las principales aportaciones de la literatura al estudio del capital de marca y su aplicación en el sector educativo, identificando qué variables determinan el capital de marca en la educación superior. Una vez establecido el modelo de capital de marca susceptible de aplicación en el sector educativo, se lleva a cabo un estudio empírico contando con una muestra cuantitativa de 416 respuestas válidas procedentes de empleados de administración y servicios de universidad. Los resultados obtenidos muestran la repercusión de cada una de las variables del capital de marca sobre dichos empleados, siendo: notoriedad de marca, imagen de marca, calidad percibida de marca y lealtad de marca.
\end{abstract}

Palabras clave: Capital de Marca; Educación Superior; Personal de Administración y Servicios; Sistemas de Ecuaciones Estructurales.

\section{[en] The importance of perceiving brand awareness and brand image among university service staff}

\begin{abstract}
This research was carried out to examine the role of brand capital in higher education through the administration and service employees. For this purpose, the main contributions of the literature related to the study of brand capital and its application in the educational sector were analyzed, identifying which variables determine brand capital in the higher education sector. Once the susceptible brand capital in the higher education sector was established, an empirical study was realised using a valid sample of 416 responses from administrative and service employees. The results show the repercussion of each variable of the brand capital on the mentioned employees, being: brand awareness, brand image, perceived quality and brand loyalty.
\end{abstract}

Keywords: Brand Capital; Higher Education; University Service Staff; Structural Equations Modeling.

Sumario. 1. Introducción. 2. El personal de administración y servicios. 3. Metodología de la investigación. 4. Resultados. 5. Conclusiones e implicaciones gerenciales.

\footnotetext{
$1 \quad$ Universidad Internacional de Valencia javier.casanoves@campusviu.es

$2 \quad$ Universidad de Valencia ines.kuster@uv.es

3 Universidad de Valencia natalia.vila@uv.es
} 
Cómo citar: Casanoves Boix, J.; Küster Boluda, I.; Vila López, N. (2017). La importancia de percibir notoriedad e imagen de marca entre el personal de servicios de universidad, Cuadernos de Estudios Empresariales, 27, 65-86.

\section{Introducción}

Dado que las universidades se encuentran operando dentro de entornos dinámicos y desafiantes, la estrategia de marketing se convierte en una prioridad para asegurar un fuerte reclutamiento y retención de estudiantes y profesores (Asaad, Melewar, Cohen, \& Balmer, 2013). Existe consenso en que la comprensión de la marca universitaria y el claro desarrollo y comunicación de la misma aporta gran valor para las instituciones educativas superiores (Duesterhaus \& Duesterhaus, 2014). Por ello, la gestión estratégica de la marca se convierte en pieza clave dentro del sector educativo y, en especial, en las instituciones educativas superiores (Rauschnabel, Krey, Babin y Ivens, 2016).

Junto a ello, la explosión de la información académica y del conocimiento avanzado está llevando a cabo importantes transformaciones en la función educativa universitaria, buscando garantizar oportunidades de formación permanente a lo largo de la vida de las personas, así como proporcionar competencias profesionales sintonizadas con los requerimientos del mercado laboral. (Bok, 2003). Con ello, el mercado de la educación superior está actualmente bien establecido como un fenómeno global, especialmente en las principales naciones de habla inglesa (Hemsley-Brown y Oplatka, 2006).

Centrando nuestra atención en el papel que juega la educación superior en España, Grijalba y López (2007) consideran que el incremento del nivel educativo alcanzado en nuestro país en los últimos años ha sido uno de los fenómenos más relevantes acaecidos. Pese a que en algunos aspectos todavía no se han logrado la cota de otros países europeos, la trayectoria experimentada ha culminado en una convergencia educativa con los países de nuestro entorno. En este contexto, argumentaron que el crecimiento del sistema universitario español ha sido clave desde comienzos de la década de los noventa. Con ello, la Universidad española ha dejado de ser una institución de carácter elitista para convertirse en un sistema que proporciona una formación de masas (Pérez y Serrano, 1998). Y dicha situación representa un fenómeno sin precedentes ya que, hasta principios de los noventa, España se había caracterizado por presentar un déficit de capital humano en todos los niveles educativos (Grijalba y López, 2007).

Con ello, Chen (2008) considera que la educación superior también proporciona un contexto interesante e importante para la investigación en marketing, ya que las instituciones de educación superior de todo el mundo se están orientando cada vez más hacia la estrategia de marketing, y los estudiantes son, cada vez más, considerados como consumidores y clientes potenciales. Más aún, Conway, Mackay y Yorke (1994) afirman que las instituciones educativas superiores han incrementado la aplicación de estrategias y políticas de marketing a fin de poder ajustarse a las demandas del entorno. Así, y de acuerdo con Küster (2012), existen ciertas evidencias que constatan que las teorías y conceptos de marketing pueden ser aplicados en el 
contexto educativo y, en especial, en el ámbito de la educación superior. Con ello, se busca obtener resultados en términos de valor, efectividad y beneficios potenciales, los cuales han sido efectivos en el mundo de los negocios a través de la consecución de ventaja competitiva y la conquista de un amplio segmento del mercado internacional.

Paralelo a ello, las marcas han ido evolucionando con el tiempo hasta convertirse en una experiencia de vida para los consumidores, habiendo adquirido una importancia emocional que se ve reflejada en la satisfacción de las personas que la consumen o compran (Camacho, 2008). Ahora bien, Küster, Vila y Aldás (2011) consideran que, en la búsqueda de nuevas formas de diferenciación que supongan la creación real de valor para el cliente, las empresas deben ser económicamente eficientes. Así, y en relación a la definición de capital de marca en la educación superior, Berry (2000) argumenta que, si bien el producto es considerado la marca principal en cuanto a productos envasados, no ocurre lo mismo en las empresas de servicios. En este caso, la empresa es la marca principal, siendo el propio servicio la piedra angular de la comercialización para hoy y mañana. Y dicha aportación, aplicada a la prestación de servicios educativos, incluye generar capital de marca en base a la fuerza de marca que transmite la propia institución educativa superior.

Más aún, Hemsley-Brown y Goonawardana (2007) consideran que el entorno económico de los últimos años ha tenido un fuerte impacto negativo en la situación ha propiciado que las instituciones de educación superior hayan recurrido a la gestión de sus marcas en su intento de prosperar, y en algunos casos para sobrevivir en el saturado mercado de la educación superior. Con ello, y teniendo en cuenta que el mercado es cada vez más complejo y competitivo, las universidades y colegios hacen uso de la gestión estratégica de marcas para hacer frente a los desafíos globales de hoy (Whisman, 2009).

En este marco, el personal de administración y servicios se convierte en pieza angular debido a que toda organización que ofrece un servicio (ya sea educativo o referido a cualquier otro sector) debe tener muy en cuenta la labor de sus empleados, lo cual es muy importante para construir marca poderosa (Lovelock y Dorfsman, 1997). Todo construye o destruye marca, y dicho proceso continuo e indefinido en el tiempo debe alimentarse de todas las acciones que la empresa (o institución educativa) dirige a sus públicos, así como las interacciones y experiencias del público con la misma (Olins, 2008). Por ello, los objetivos del presente artículo pasan por (1) analizar qué se entiende por capital de marca y cuáles son sus variables determinantes en el sector educativo, y (2) descifrar a nivel empírico cuáles son las variables más destacables del capital de marca educativo por parte del personal de administración y servicios.

Con ello, Camisón (1998) argumenta que las presiones competitivas a las que están sometidas las universidades hacen que éstas deban ser sensibles al concepto de calidad en administración y prestación de servicios. Por tanto, las universidades deben adaptarse a las nuevas formas de gestión, donde prime el trato con el cliente y el buen hacer de sus empleados, siendo el personal de gestión parte responsable de transmitir, dentro de su puesto de trabajo, dicha estrategia a los clientes (Deem y Brehony, 2005). Más aún, el sistema educativo se está globalizando de tal modo que cualquier estudiante procedente de cualquier país puede cursar parte o todos sus estudios en universidades extranjeras. Con ello, los empleados de gestión deben estar capacitados para entender las peticiones de dichos clientes e intercambiar 
impresiones en distintos idiomas (Altbach y Knight, 2007). En definitiva, y como señala Coleman, Campbell, Hobson, McPartland, Mood, Weinfeld y York (1966), el servicio prestado dentro del sistema universitario deben ser uno de los pilares que marquen la diferencia.

Así, y en el escenario descrito, la realización de la presente investigación supone un avance científico en la disciplina de marketing puesto que hasta la fecha, aunque se han realizado investigaciones específicas del capital de marca y las variables que lo componen (Farquhar, 1989; Aaker, 1992; Keller, 1993; Faircloth, Capella y Alford, 2001; Yoo y Donthu, 2001; Delgado y Munuera, 2002; Buil, Martínez y De Chernatony, 2010), no existen investigaciones que analicen exhaustivamente la percepción del capital de marca a través de opiniones de personal administrativo y de servicios en instituciones educativas de carácter superior en España (Flavián y Lozano, 2002) Asimismo, puede servir de ayuda a los directivos universitarios puesto que pueden descifrar las claves en cuanto a las opiniones de su personal de administración y servicios (PAS) con respecto al capital de marca de la propia institución y generar, con ello, estrategias adecuadas para mantenerlo o mejorarlo.

\section{El personal de administración y servicios}

Para entender correctamente este de agente implicado en las instituciones educativas superiores, y siendo considerado también como cliente interno, correspondiente a los empleados de servicios, se ha dividido la información encontrada en la literatura en dos conceptos clave, siendo: (1) importancia del personal de administración y servicios, y (2) la percepción del capital de marca entre el personal de administración y servicios. A continuación se detalla cada uno de ellos.

\section{a) Importancia del personal de administración y servicios}

En primer lugar, y en relación a la importancia de los empleados de servicios, o también definido como personal de administración y servicios (PAS), Lovelock y Dorfsman (1997) consideran que toda organización (lucrativa o no) debe tener muy en cuenta la estrategia desarrollada en torno al servicio que ofrecen. Y ello, extrapolado al ámbito universitario, se adecuaría en torno al personal que realiza las tareas de administración y servicios.

Por su parte, Edo, Puig y Zornoza (1999) creen que las universidades deben crear modelos organizacionales de calidad orientados al cliente. Así, exponen la problemática en torno a la medición de la calidad del servicio prestado en las universidades, ya sea a través de la docencia impartida como por las distintas tareas de administración y gestión que pueden desempeñarse para cubrir las necesidades globales del cliente. Además, exponen la importancia de medición del grado de implicación de los empleados de servicios, de cara a entender la problemática existente en ciertas universidades.

Por su parte, Zeithaml, Bitner, De Lara Choy, Hirschfeld y Becerril (2002) señalan la importancia de disponer de un adecuado personal de administración y servicios como medio para poder satisfacer completamente las necesidades de los clientes. Por tanto, Ferrer (2005) defiende la importancia de la percepción del cliente de aquellos 
elementos determinantes de la calidad al servicio educativo, tanto a nivel de características del propio servicio como de habilidades profesionales de los empleados que trabajan en la misma. Así, la figura del personal de administración y servicios se hace imprescindible dentro de la organización universitaria.

Como señalan Hunger, Sánchez, Mejía y Hunger (2007) el personal de servicio actúa como elemento visible durante la vida estudiantil del cliente final. No en vano, Douglas, McClelland y Davies (2008) acentúan que, entre las variables determinantes que generan alta calidad percibida para el estudiante, se conciben aquellas relacionadas con el personal prestador de servicios, ya sean tareas de dirección, gestión, administración o docencia. Con ello, Butin (2010) introduce el término engagement entre universidad y cliente-alumno, a través del cual enfatiza la importancia de los empleados de gestión universitaria en generar buenas relaciones con los pupilos, debiendo sentirse íntegramente comprometidos con la organización universitaria.

Más recientemente, Sultan y Yin Wong (2012) exponen que la calidad del servicio educativo se compone de tres factores, siendo: (1) la confianza del cliente con la marca, (2) la satisfacción del cliente con el servicio recibido y (3) la imagen que la universidad transmite al exterior.

Por tanto, parte de esta imagen la transmiten los empleados de servicios, los cuales están en contacto directo y habitual con los estudiantes, y son los que mejor pueden resolver aquellos problemas relacionados con sus quehaceres diarios. En el marco descrito, la percepción que el personal disponga de la institución en la que trabaja afectará al desempeño de su trabajo.

En suma, Clotfelter (2014) explica que, parte del coste tangible e intangible de las instituciones educativas por escalar posiciones en los rankings globales pasa por dar un buen servicio a todos los niveles, siendo por tanto la labor del personal de administración y gestión un elemento clave en la universidad para conseguir dichos objetivos estratégicos.

\section{b) La percepción del capital de marca entre el personal de administración y servicios}

En segundo lugar, y en relación a la percepción del capital de marca entre el personal de servicios, éste ha sido estudiado por diversos investigadores desde los años 60 hasta la actualidad, siendo considerado como un activo muy valioso para transmitir los servicios educativos que las marcas universitarias ofrecen al mercado (McAlexander, Koenig y Schouten, 2006). Además, tal y como se ha comentado anteriormente, también se les considera como clientes internos de la organización, siendo por tanto de vital importancia tratar de descifrar sus percepciones sobre capital de marca educativo que exponemos en la presente investigación.

Así pues, y después de revisar las siete propuestas principales sobre modelos de capital marca efectuadas por la literatura (Farquhar, 1989; Aaker, 1992; Keller, 1993; Faircloth, Capella y Alford, 2001; Yoo y Donthu, 2001; Delgado y Munuera, 2002; Buil, Martínez y De Chernatony, 2010), se han considerado cuatro elementos que, compartidos por los autores y teniendo en cuenta la importancia otorgada en estudios previos, entendemos que su aportación al capital de marca es clave. Estos son: (1) notoriedad de marca, (2) imagen de marca, (3) calidad percibida y (4) lealtad de marca. Y, junto a dichas aportaciones, se han revisado una serie de investigaciones que 
soportan cada uno de los elementos del capital de marca en su aplicación al sector educativo, representadas a través de la Tabla 1.

Tabla 1. Principales elementos del capital de marca en la educación superior

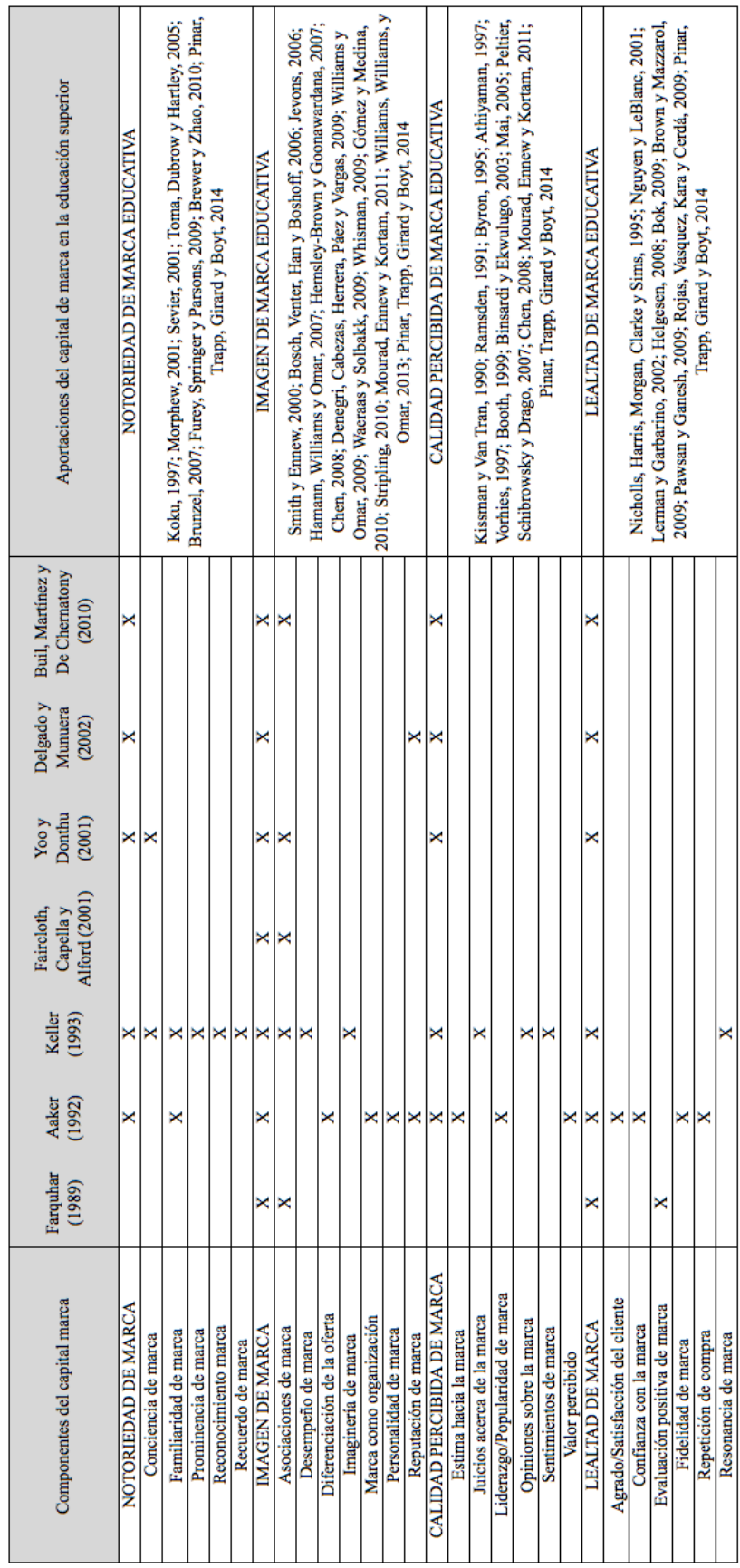

Fuente: Elaboración propia 


\section{H1. Notoriedad de marca}

La notoriedad de marca consiste en el desempeño del reconocimiento y recuerdo de la marca, caracterizada por la forma en que los consumidores adoptan sus decisiones (Aaker, 1992).

En el ámbito concreto de la educación, diversos autores (Toma, Dubrow y Hartley, 2005; Brunzel, 2007; Furey, Springer y Parsons, 2009; Brewer y Zhao, 2010; Pinar, Trapp, Girard y Boyt, 2014) constatan en sus estudios que la notoriedad de marca en la educación superior es una variable muy importante para las universidades, de cara a generar mayor preferencia de consumo en servicios educativos y, con ello, incremento de ventas. Es decir, una mayor notoriedad de marca, supondrá un mayor capital de marca.

Con ello, y en relación a la notoriedad de marca y el personal de servicios, Mourad, Ennew y Kortam (2011) señalan que éste percibe mayor notoriedad a medida que la institución consigue más reconocimiento de marca por parte de la sociedad. Por ello, se debe explotar al máximo los recursos que tiene la institución. En la misma línea, Zlotkowski (1998) considera que el hecho de comprometer a todo el capital humano (sobretodo a los empleados de servicios) y generar una comunidad con el alumnado ayuda a incrementar la notoriedad de marca educativa. Así, las universidades públicas y privadas deben invertir parte de su presupuesto en formar a dichos empleados y adquirir los instrumentos necesarios para integrarlos a todos en una misma comunidad.

A la vista de lo expuesto en líneas previas, es posible plantear la primera hipótesis de investigación.

H1. La percepción sobre notoriedad de marca influye en la percepción del capital de marca del personal de administración y servicios en las instituciones de educación superior.

\section{H2. Imagen de marca}

Las imágenes mentales que se pueda hacer un consumidor de una marca están íntegramente relacionadas con el desempeño y el esfuerzo que ésta realice en su creación de valor, los cuales están íntegramente relacionados con las asociaciones, diferenciación de producto e imagen y personalidad que transmite la marca (Keller, 1993).

En el ámbito concreto de la educación, diversos autores (Jevons, 2006; Hamann, Williams y Omar, 2007; Hemsley-Brown y Goonawardana, 2007; Chen, 2008; Denegri, Cabezas, Herrera, Páez y Vargas, 2009; Williams y Omar, 2009; Waeraas y Solbakk, 2009; Whisman, 2009; Gómez y Medina, 2010; Stripling, 2010; Mourad, Ennew y Kortam, 2011; Williams, Williams, y Omar, 2013; Pinar, Trapp, Girard y Boyt, 2014) constatan que la imagen de marca en las universidades públicas y privadas es un atractivo muy valioso para las organizaciones, debiéndose realizar mediciones eficaces y rápidas de la imagen trasmitida por los públicos objetivos puesto que dicha percepción puede resultar diferente en ambos ámbitos. Esta imagen, en la medida que mejore, conllevará aumentos del capital de marca de la institución universitaria cuya imagen se vea favorecida.

Con ello, y en relación a la imagen de marca y el personal de servicios, McAlexander, Koenig y Schouten (2006) consideran que la imagen de marca de una universidad se maximiza a través de la construcción de relaciones sostenibles en el tiempo entre los trabajadores de la propia institución y la comunidad estudiantil. Edo, Puig y Zornoza (1999) consideran que generar modelos de calidad del servicio 
orientado a los clientes, en los que el personal de administración y servicios tienen un papel fundamental, ayuda a mejorar la imagen de la marca universitaria y, con ello, se mejora el valor capital de la misma. Y, Sung y Yang (2008) añaden que si una universidad consigue generar un modelo de marca basado en personalidad fuerte, prestigio externo y reputación hará que ésta mejore su imagen de marca con respecto a sus estudiantes, aunque generalmente será percibida en mayor medida por parte de los propios trabajadores (profesores y personal de servicios) ya que éstos tienen más contacto y destinan más tiempo a la marca a lo largo de su vida útil como clientes.

A la vista de lo expuesto en líneas previas, es posible plantear la segunda hipótesis de investigación.

H2. La percepción sobre imagen de marca influye en la percepción del capital de marca del personal de administración y servicios en las instituciones de educación superior.

\section{H3. Calidad percibida de marca}

La calidad percibida se basa en las actitudes hacia las marcas, siendo evaluaciones generales que forman parte de la elección de consumo por parte de los consumidores. Y estas actitudes dependen, generalmente, de los atributos y beneficios específicos de la marca (Wilkie, 1994). La calidad percibida es, por tanto, un juicio subjetivo, por lo que la percepción de calidad puede corresponderse con calidad real, o no (Chieng-Huang y Kao, 2004).

En el ámbito concreto de la educación, diversas investigaciones (Binsardi y Ekwulugo, 2003; Mai, 2005; Peltier, Schibrowsky y Drago, 2007; Chen, 2008; Mourad, Ennew y Kortam, 2011; Pinar, Trapp, Girard y Boyt, 2014) constatan que la calidad percibida en la educación superior, privada y pública, es una variable a tener muy en cuenta también, ya que de ella depende que las instituciones educativas obtengan mayor satisfacción por parte de sus usuarios. La calidad, por tanto, es otra fuente clave en la configuración del capital de la marca de la institución universitaria, en tanto en cuanto mayor calidad percibida, se traducirá en mayor capital de marca.

Con ello, y en relación a la calidad percibida de marca y el personal de servicios, Harvey y Green (1993) consideran que la calidad percibida en la educación superior es un concepto complejo y multifacético y una única definición adecuada de la calidad es deficiente. Con ello, el personal de servicios tiene un papel fundamental en la maximización de dicha percepción. Y, diversos investigadores (Miquel, Bigné, Sánchez, Moliner y Vallet, 1995; Lozier y Teeter, 1996; Schargel, 1996) enfatizan la necesidad de determinar la calidad de un servicio administrativo basándose en indicadores objetivos (elementos formales) pero también en indicadores subjetivos (basados en la calidad percibida del servicio). Y todo ello puede discernir dependiendo del ámbito público o privado de la propia universidad.

A la vista de lo expuesto en líneas previas, es posible plantear la tercera hipótesis de investigación.

H3. La percepción sobre calidad percibida de marca influye en la percepción del capital de marca del personal de administración y servicios en las instituciones de educación superior.

\section{H4. Lealtad de marca}

La lealtad de marca se asocia con el comportamiento del cliente hacia su repetición de compra, traduciendo dicha lealtad como la disposición a pagar mayores precios por la misma, menores costes de servicio y el incremento de nuevos clientes a través de la 
atracción (Aaker, 1996). Cuando un cliente es fiel, suele comprar la misma marca con frecuencia, ocasionando una mayor participación al mercado (Assael, 1998).

En el ámbito concreto de la educación, trabajos diversos (Nguyen y LeBlanc, 2001; Lerman y Garbarino, 2002; Bok, 2003; Helgesen, 2008; Brown y Mazzarol, 2009; Pawsan y Ganesh, 2009; Rojas, Vasquez, Kara y Cerdá, 2009; Pinar, Trapp, Girard y Boyt, 2014) constatan la especial relevancia que tiene la lealtad de marca en el sector educativo superior, lo que implica que dicha variable es muy importante a la hora de generar capital de marca educativo. Públicos más leales, contribuyen a incrementar el capital de marca.

Con ello, y en relación a la imagen de marca y el personal de servicios, Edo, Puig y Zornoza (1999) añaden que generar modelos de calidad del servicio orientados a los clientes derivan en un incremento de fidelidad hacia la marca por parte del capital humano interno (profesores y personal de servicios) y externo (alumnos), además de la propia mejora de la imagen de marca comentada anteriormente. Y, Hennig, Langer y Hansen (2001) añaden que las tasas de matrícula, la flexibilidad de la organización y el compromiso del personal interno con su propia institución hacen que sus estudiantes sientan mayor lealtad a su universidad en comparación con los propios empleados, debido a que, generalmente, necesitan otro tipo de incentivos (económicos y sociales) para incrementar su fidelidad hacia la marca educativa.

A la vista de lo expuesto en líneas previas, es posible plantear la cuarta hipótesis de investigación.

H4. La percepción sobre lealtad de marca influye en la percepción del capital de marca del personal de administración y servicios en las instituciones de educación superior.

A la vista de lo expuesto, la Figura 1 muestra nuestra propuesta de modelo teórico diseñado para defender la presente investigación.

Figura 1. Modelo teórico propuesto en base al rol del personal de administración y servicios

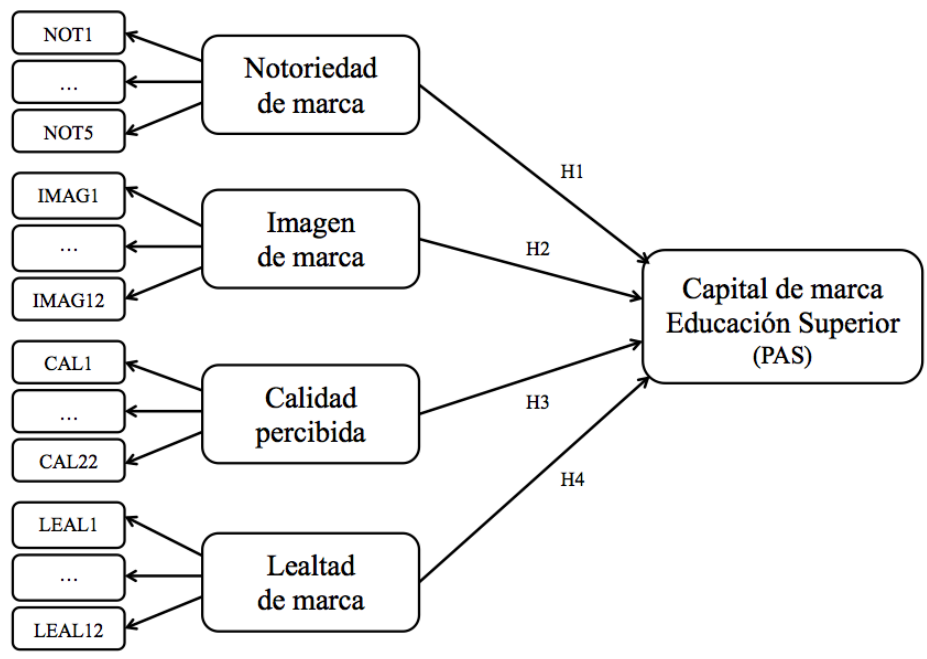

Fuente: Elaboración propia 


\section{Metodología de la investigación}

Para alcanzar los objetivos descritos y contrastar las hipótesis planteadas, se llevó a cabo un estudio cuantitativo en una ciudad española, Valencia (España), porque se considera que ha apostado y apuesta por la educación universitaria, tanto pública como privada.

Según el estudio desarrollado por el Instituto Valenciano de Investigaciones Económicas (2008), el 60\% de las ofertas laborales de la próxima década serán para aquellos que posean estudios superiores, por lo que la educación superior aumentará en 13 puntos porcentuales la probabilidad de trabajar de un joven respecto a otro que sólo cuenta con estudios obligatorios. Con ello, Valencia es percibida por el mercado estudiantil como una gran sede educativa gracias al asentamiento de tres centros educativos superiores de ámbito público y siete privados (tanto universitarios como centros adscritos) en los últimos años, que han proyectado la imagen de la ciudad a nivel nacional, contribuyendo a su notoriedad y al conocimiento por parte del público en general. Por ello, se ha optado por incluir en el presente estudio una muestra de dos universidades públicas y seis privadas instauradas en Valencia, todas consideradas referentes tanto en la Comunidad Valenciana como a nivel nacional (Gómez y Gutiérrez, 2001).

Para cuantificar la población objetivo se utilizaron datos oficiales de Facultades relacionadas con el ámbito de Economía de ocho instituciones educativas superiores. La recogida de información se realizó a través de un cuestionario en formato digital y papel distribuido a un 262 empleados de servicios de universidad pública y 169 de universidad privada. Se empleó un muestreo no probabilístico por conveniencia. Tras la recogida y depuración de la información, se obtuvieron un total de 416 encuestas válidas.

Como datos destacables del perfil de la muestra, cerca del $60,6 \%$ de los encuestados procede del ámbito educativo público, siendo el otro $39,4 \%$ de privado, lo cual indica que la muestra es lo suficientemente heterogénea, debido a la similar cantidad de respuestas de ambos ámbitos. Además, el intervalo de edad de los encuestados se sitúa en torno a los 43 años, destacando que se albergan muchas más respuestas de mujeres que hombres (67\% con respecto al 32\%). Destacar, con ello, el alto número de trabajadoras mujeres con respecto al bajo número de hombres. Y, con respecto a las nacionalidades de los distintos participantes, destacar que el 97,36\% de respuestas provienen de empleados españoles, mientras que el otro 1,68\% procede de trabajadores de Argentina, Bélgica, Colombia, Francia, Marruecos y Rumanía. Finalmente, destacar, como aspecto positivo, la gran participación obtenida y, con ello, el bajo porcentaje de encuestados que han omitido responder a estas preguntas de clasificación $(0,96 \%)$.

Por su parte, y en lo relativo a antigüedad, el $90 \%$ de respuestas se sitúa en torno a más de 10 años y con contrato laboral a tiempo completo. A su vez, la inmensa mayoría PAS trabaja más de 300 horas anuales y el salario bruto medio mensual se sitúa en un rango inferior entre 1.000 y 2.000 euros. Por su parte, y en relación al nivel de estudios, destacar que más del $75 \%$ de la plantilla poseen estudios de bachillerato, formación profesional o estudios universitarios, siendo requisitos mínimos necesarios para acceder a un puesto de trabajo. Además, más del $30 \%$ de respuestas provienen de personal dedicado a la administración, mientras que un $15 \%$ proviene de técnicos de gestión. Todo ello indica que las respuestas recibidas por los grupos 
de mayoría de encuestados son de calidad, ya que proviene de personal que tiene amplia experiencia y buenos conocimientos sobre el funcionamiento y el músculo financiero de su institución.

Se emplearon escalas Likert de grado 5 para medir los conceptos de (1) notoriedad de marca, (2) imagen de marca, (3) calidad percibida de marca y (4) lealtad de marca. Dichas escalas están basadas en las escalas de medición propuestas por Aaker (1992) y Keller (1993).

Finalmente, las técnicas para el análisis de datos están basadas en la Estadística Descriptiva y el Análisis Multivariante, recurriéndose como herramienta de trabajo al SPSS v19 para Windows para las técnicas descriptivas de los datos y al EQS 6.2 para ejecutar las técnicas multivariantes. El procesamiento estadístico de los datos seguido en este estudio supone la aplicación de distintos métodos de análisis en función de la información que se desea obtener, distinguiendo entre: (1) descripción y clasificación de los datos, y (2) contraste de hipótesis.

\section{Resultados}

Previo al contraste de hipótesis, se analizaron las características psicométricas del instrumento de medida. Para ello, se dividió la información en (1) un análisis de la calidad de los ítems y (2) la validación de las escalas, la cual se muestra en la tabla número 2 .

La fiabilidad se comprobó mediante tres métodos de análisis, siendo: (1) a de Cronbach (CA) obteniendo, en todos los casos, valores superiores a 0,7 (Nunnally y Bernstein, 1994); (2) análisis de fiabilidad compuesta (CR) obteniendo también valores superiores 0,7 (Carmines y Zeller, 1979); y (3) análisis de la varianza extraída promedio (AVE), donde los resultados fueron cercanos, iguales o superiores a 0,5 (Fornell y Larcker, 1981).

Además, las medidas de bondad de ajuste de los modelos presentan valores aceptables $(\mathrm{NFI}=0,73 ; \mathrm{NNFI}=0,82 ; \mathrm{CFI}=0,83 ; \mathrm{IFI}=0,83)$ ya que están cerca de 0,9 (Hair, Black, Babin, Anderson y Tatham, 2005) y el indicador RMSEA= 0,06 también presenta un buen ajuste, siendo su valor inferior a 0,08 (Steinger, 1990), sugiriendo que el modelo estructural encaja bien con la estructura de datos. Y, aunque no todos los valores de las cargas factoriales por separado son superiores a 0,6 (Bagozzi y Yi, 1988), bien es cierto que el promedio de las cargas factoriales estandarizadas $(\bar{X})$ son cercanos o superiores a 0,7 (Currás, 2007), constatándose así la validez convergente del modelo propuesto. 
Tabla 2. Fiabilidad y validez convergente

\begin{tabular}{|c|c|c|c|c|c|c|c|}
\hline FACTOR & INDICADOR & CARGA & $t$ ROBUSTO & $\bar{X}$ CARGA & $\mathrm{CA}$ & CR & AVE \\
\hline \multirow{5}{*}{$\begin{array}{c}\text { NOT. } \\
\text { Notoriedad } \\
\text { de marca }\end{array}$} & NOT1 & $0,44 * * *$ & \begin{tabular}{|l|}
6,79 \\
\end{tabular} & \multirow{5}{*}{0,74} & \multirow{5}{*}{0,85} & \multirow{5}{*}{0,86} & \multirow{5}{*}{0,57} \\
\hline & NOT2 & $0,70^{* * *}$ & 11,29 & & & & \\
\hline & NOT3 & $0,94 * * *$ & 18,17 & & & & \\
\hline & NOT4 & $0,90 * * *$ & 17,55 & & & & \\
\hline & NOT5 & $0,71^{* * *}$ & 13,39 & & & & \\
\hline \multirow{11}{*}{$\begin{array}{l}\text { IMAG. } \\
\text { Imagen de } \\
\text { marca }\end{array}$} & IMAG1 & $0,67 * * *$ & 13,38 & \multirow{11}{*}{0,70} & \multirow{11}{*}{0,91} & \multirow{11}{*}{0,91} & \multirow{11}{*}{0,50} \\
\hline & IMAG2 & $0,72^{* * *}$ & 14,78 & & & & \\
\hline & IMAG3 & $0,81^{* * *}$ & 16,13 & & & & \\
\hline & IMAG4 & $0,73 * * *$ & 16,01 & & & & \\
\hline & IMAG5 & $0,58 * * *$ & 9,70 & & & & \\
\hline & IMAG6 & $0,76^{* * *}$ & 14,70 & & & & \\
\hline & IMAG7 & $0,75^{* * *}$ & 15,21 & & & & \\
\hline & IMAG8 & $0,44 * * *$ & 7,53 & & & & \\
\hline & IMAG9 & $0,74^{* * *}$ & 15,05 & & & & \\
\hline & IMAG10 & $0,68^{* * *}$ & 12,51 & & & & \\
\hline & IMAG11 & $0,72^{* * *}$ & 13,09 & & & & \\
\hline \multirow{21}{*}{$\begin{array}{c}\text { CAL. } \\
\text { Calidad } \\
\text { percibida } \\
\text { de marca }\end{array}$} & CAL1 & $0,78^{* * *}$ & 15,45 & \multirow{21}{*}{0,72} & \multirow{21}{*}{0,96} & \multirow{21}{*}{0,96} & \multirow{21}{*}{0,52} \\
\hline & CAL2 & $0,58^{* * *}$ & 11,08 & & & & \\
\hline & CAL3 & $0,79 * * *$ & 15,86 & & & & \\
\hline & CAL4 & $0,80^{* * *}$ & 17,50 & & & & \\
\hline & CAL5 & $0,68^{* * *}$ & 13,11 & & & & \\
\hline & CAL6 & $0,68^{* * *}$ & 13,43 & & & & \\
\hline & CAL7 & $0,65^{* * *}$ & 11,14 & & & & \\
\hline & CAL8 & $0,72^{* * *}$ & 14,84 & & & & \\
\hline & CAL9 & $0,70^{* * *}$ & 14,49 & & & & \\
\hline & CAL10 & $0,67 * * *$ & 13,08 & & & & \\
\hline & CAL11 & $0,68^{* * *}$ & 13,03 & & & & \\
\hline & CAL12 & $0,79 * * *$ & 16,82 & & & & \\
\hline & CAL13 & $0,82 * * *$ & 15,69 & & & & \\
\hline & CAL14 & $0,73^{* * *}$ & 14,29 & & & & \\
\hline & CAL15 & $0,78^{* * *}$ & 19,20 & & & & \\
\hline & CAL16 & $0,67 * * *$ & 13,25 & & & & \\
\hline & CAL17 & $0,75^{* * *}$ & 15,73 & & & & \\
\hline & CAL18 & $0,70^{* * *}$ & 12,51 & & & & \\
\hline & CAL19 & $0,59 * * *$ & 9,32 & & & & \\
\hline & CAL20 & $0,79 * * *$ & 14,65 & & & & \\
\hline & CAL21 & $0,76^{* * *}$ & 13,01 & & & & \\
\hline \multirow{11}{*}{$\begin{array}{l}\text { LEAL. } \\
\text { Lealtad de } \\
\text { marca }\end{array}$} & LEAL1 & $0,76^{* * *}$ & 13,09 & \multirow{11}{*}{0,79} & \multirow{11}{*}{0,95} & \multirow{11}{*}{0,95} & \\
\hline & LEAL2 & $0,84 * * *$ & 14,92 & & & & \\
\hline & LEAL3 & $0,85^{* * *}$ & 17,58 & & & & \\
\hline & LEAL4 & $0,89 * * *$ & 20,93 & & & & \\
\hline & LEAL5 & $0,88^{* * *}$ & 19,74 & & & & \\
\hline & LEAL6 & $0,70^{* * *}$ & 13,10 & & & & 0,63 \\
\hline & LEAL7 & $0,85^{* * *}$ & 19,12 & & & & \\
\hline & LEAL8 & $0,69^{* * *}$ & 11,58 & & & & \\
\hline & LEAL9 & $0,79 * * *$ & 14,36 & & & & \\
\hline & LEAL10 & $0,79 * * *$ & 14,14 & & & & \\
\hline & LEAL11 & $0,70^{* * *}$ & 12,63 & & & & \\
\hline & CM1 & $0,58^{* * * *}$ & 10,01 & & & & \\
\hline CM. Capital & CM2 & $0,69 * * *$ & 15,13 & 067 & 078 & 077 & 0,46 \\
\hline de marca & CM3 & $0,84 * * *$ & 17,14 & $0,0 \prime$ & 0,10 & & \\
\hline & CM4 & $0,57 * * *$ & 8,16 & & & & \\
\hline
\end{tabular}

$\mathrm{N}=416 ;{ }^{* * *} \mathrm{p}<0,01 ; * * \mathrm{p}<0,05 ; * \mathrm{p}<0,1 ;$ Satorra-Bentler $\mathrm{c}^{2}(\mathrm{p})=2.823,1(0.0000), \mathrm{df}=1.259$;

$\mathrm{NFI}=0,73 ; \mathrm{NNFI}=0,82 ; \mathrm{CFI}=0,83 ; \mathrm{IFI}=0,83 ; \mathrm{RMSEA}=0,06$ 
Finalmente, y mediante un modelo de ecuaciones estructurales basado en el método de máxima verosimilitud robusto, se llevó a cabo el contraste de hipótesis, tal y como se muestra en la Tabla siguiente.

Tabla 3. Contraste de hipótesis

\begin{tabular}{|l|l|l|l|l|}
\hline Hipótesis & Relación Estructural & $\beta$ Estand. & T Robusto & Criterio \\
\hline $\mathrm{H}_{\text {(PAS) }}$ & $\begin{array}{l}\text { Percepción Notoriedad de Marca --> } \\
\text { Percepción Capital de Marca }\end{array}$ & $0,242^{* * *}$ & 4,43 & Aceptada \\
\hline $\mathrm{H}_{\text {(PAS) }}$ & $\begin{array}{l}\text { Percepción Imagen de Marca --> Per- } \\
\text { cepción Capital de Marca }\end{array}$ & $0,496^{* * *}$ & 3,39 & Aceptada \\
\hline $\mathrm{H}_{\text {(PAS) }}$ & $\begin{array}{l}\text { Percepción Calidad Percibida --> Per- } \\
\text { cepción Capital de Marca }\end{array}$ & 0,232 & 1,04 & Rechazada \\
\hline $\mathrm{H}_{(\mathrm{PAS})}$ & $\begin{array}{l}\text { Percepción Lealtad de Marca --> Per- } \\
\text { cepción Capital de Marca }\end{array}$ & 0,094 & Rechazada
\end{tabular}

$\mathrm{N}=416 ; * * * \mathrm{p}<0,01 ; * * \mathrm{p}<0,05 ; * \mathrm{p}<0,1$

Satorra-Bentler $\mathrm{c}^{2}(\mathrm{p})=2.823,1(0.0000), d f=1.259 ; \mathrm{NFI}=0,73 ; \mathrm{NNFI}=0,82 ; \mathrm{CFI}=0,83 ; \mathrm{IFI}=0,83$; RMSEA $=0,06$

Como se puede observar, los resultados sugieren que el modelo diseñado en esta investigación aplicado al personal de administración y servicios resulta satisfactorio para explicar 2 de las 4 hipótesis planteadas. Así, se demostró una relación positiva entre las percepciones de notoriedad de marca e imagen de marca y capital de marca, aceptando la hipótesis primera (H1PAS: $\beta=0,242 ; \mathrm{p}<0,01$ ) y la hipótesis segunda (H2PAS: $\beta=0,496 ; p<0,01)$. Es decir, se irá consolidando una percepción favorable hacia el capital de marca a medida que se vayan construyendo percepciones positivas hacia la notoriedad y la imagen de la marca educativa. Pero, en lo relativo a la hipótesis tercera (H3PAS: $\beta=0,232$; ns) y la hipótesis cuarta (H4PAS: $\beta=0,094$; ns) cabe destacar que no se demostró una relación positiva entre la percepción de calidad percibida de marca, lealtad de marca y capital de marca por parte de los 416 empleados de administración y servicios encuestados.

\section{Conclusiones e implicaciones gerenciales}

Como conclusiones generales de los resultados obtenidos en el contraste de hipótesis, destacar que el modelo global planteado demostró una relación positiva y directa entre las percepciones de notoriedad de marca (tal y como concluyeron en sus investigaciones Mourad, Ennew y Kortam, 2011; Pinar, Trapp, Girard y Boyt, 2014) e imagen de marca (Webb y Jagun, 1997; Cubillo, Sánchez y Cerviño, 2006; Cervera, Schlesinger, Mesta y Sánchez, 2012) y capital de marca. Por el contrario, no se demostró un vínculo afirmativo entre la percepción de calidad percibida de marca, lealtad de marca y capital de marca por parte del personal de administración y servicios de universidad.

Fruto de los resultados y conclusiones alcanzadas es posible plantear cuatro implicaciones gerenciales. En primer lugar, y con respecto a la notoriedad de marca, 
los resultados muestran que las instituciones educativas superiores están realizando un gran trabajo de posicionamiento de marca, planteando acciones de marketing dirigidas a sus clientes internos. Además, dicha variable es percibida como la más importante por el personal de servicios de la universidad. Tales acciones han conseguido generar gran boca oído entre su capital humano, alto conocimiento y buenas opiniones sobre el centro e interesantes recomendaciones en caso de que dichos clientes decidieran realizar unos estudios de grado o posgrado. Aun así, se recomienda a las mismas crear nuevas campañas de branding (ya sea en el entorno tradicional o digital) con el objetivo de mejorar los resultados obtenidos en el cuestionario y dar mayor visibilidad a los servicios educativos que ofrece.

En segundo lugar, y centrándonos en la imagen de marca, los resultados refuerzan el hecho de que el personal de servicios tiene una buena opinión sobre el capital que tiene la marca en cuanto a precio-calidad, transmisión de confianza, admiración y crecimiento profesional en torno a sus estudiantes. Además, muchos de ellos consideran que otras personas a las que admiran les gustaría trabajar en sus centros, lo que nos hace pensar que se han conseguido plasmar los mensajes de buen hacer entre su público interno. Pero, bien es cierto que se han generado puntuaciones a la baja en cuanto a prestaciones especiales o mantenimiento y servicio proporcionado. Por ello, se considera debería mejorarse la apuesta por dar un mejor servicio a sus empleados en cuanto a instalaciones y mantenimiento, sin dejar de lado el buen hacer que están realizando con el resto de acciones que proporcionan una alta imagen de marca.

En tercer lugar, focalizándonos en la calidad percibida de marca, el análisis estructural nos ha permitido concluir que los resultados no muestran un efecto significativo de dicha variable sobre el capital de marca. Ello puede deberse a que el personal de servicios no está completamente satisfecho con la calidad que le aporta su institución y, por tanto, existe falta de consenso en cuanto a satisfacción de las necesidades. Aunque bien es cierto que se ha se ha conseguido crear un buen equipo de trabajo, con relaciones interprofesionales sólidas y estables entre grupos de trabajo, consiguiendo además que éstos se preocupen por los alumnos y por sus compañeros de trabajo, cabe destacar que la preocupación que tienen ciertos profesionales por la escasa contraprestación económica percibida hace que la calidad percibida sea un elemento que puntúa negativamente en el capital de marca de ambas instituciones.

En cuarto y último lugar, y en cuanto la lealtad de marca, los resultados tampoco muestran un efecto significativo de dicha variable sobre el capital de marca. Ello puede deberse a que el personal de administración y servicios no tendría problema en cambiar de institución, siempre y cuando la nueva le ofrezca prestaciones más interesantes. Además, muchos de ellos no se identifican con los valores de la propia institución educativa, teniendo un bajo sentido de pertenencia a la misma y apenas simpatizando con sus compañeros de trabajo a nivel de valores humanos y de cultura corporativa. Por ello, se considera que los gestores educativos deben apostar más por generar espíritu de equipo a través de actividades que ayuden a desarrollar, de forma efectiva, las habilidades de integración cultural y organizativa, así como elaborar planes de mejora individual que hagan que su capital humano incremente su fidelidad hacia la propia institución.

Por su parte, y hablando de limitaciones de la investigación, destacar que existe un quinto elemento (otros activos de la marca, o también llamado comportamiento del mercado) susceptible de ser incorporado como variable del capital de marca; elemento que ha sido defendido por diversos autores (Farquhar, 1989; Aaker, 1992; 
Faircloth, Capella y Alford, 2001; Delgado y Munuera, 2002). Otra cuestión a considerar es la referida al tamaño de la muestra, considerando que si se hubiera ampliado la misma a más instituciones educativas superiores (públicas y privadas) habrían aumentado la calidad de la presente investigación. Así, la composición de la muestra también ha actuado como una limitación del estudio, ya que se ha realizado una técnica íntegramente cuantitativa, considerando que si se hubiera complementado con un estudio cualitativo posiblemente habría mejorado la calidad de la presente investigación.

Finalmente, y hablando de futuras líneas de investigación, resultaría especialmente interesante incluir otros colectivos colaboradores con el ámbito de la educación superior a nivel interno (los profesores o el propio equipo directivo) y externo (los estudiantes o el público en general), lo cuales se consideran son importantes para aumentar la calidad de la medición del capital de marca de una institución educativa, ya sea de carácter público o privado.

\section{Referencias}

Aaker, D.A. (1992). The value of brand equity. Journal of business strategy, Vol. 13(4), pp. 27-32.

Aaker, D.A. (1996). Measuring brand equity across products and markets, California Management Review, Vol. 38, Spring, pp. 102-20.

Altbach, P.G., \& Knight, J. (2007). The internationalization of higher education: Motivations and realities. Journal of studies in international education, Vol. 11(3-4), pp. 290-305.

Asaad, Y., Melewar, T.c., Cohen, G., \& Balmer, J. (2013). Universities and export market orientation: An exploratory study of UK post-92 universities. Marketing Intelligence \& Planning, 31(7), 838-856.

Assael, H. (1998). Consumer Behavior and Marketing Action, Cincinnati, OH: South-Western.

Bagozzi, R.p., \& Yi, Y. (1988). On the evaluation of structural equation models. Journal of the academy of marketing science, 16(1), 74-94.

Berhard, P. (2002). La formación en el uso de la información: Una ventaja en la enseñanza superior, Situación actual. Canales de Documentación, Vol 5, pp. 409-435.

Berry, L.L. (2000). Cultivating service brand equity. Journal of the Academy of marketing Science, Vol. 28(1), pp. 128-137.

Binsardi, A., \& Ekwulugo, F. (2003). International marketing of British education: research on the students' perception and the UK market penetration, Marketing Intelligence \& Planning, Vol. 21 No. 5, pp. 318-27.

Bok, D. (2003). Universities in the Marketplace. Princeton and Oxford: Princeton University Press.

Brewer, A., \& Zhao, J. (2010). The impact of a pathway college on reputation and brand awareness for its affiliated university in Sydney. International Journal of Educational Management, 24(1), 34-47.

Brown, R.m., \& Mazzarol, T.w. (2009). The importance of institutional image to student satisfaction and loyalty within higher education. Higher Education, Vol. 58(1), pp. 81-95.

Brunzel, D.L. (2007): Universities sell their brands, Journal of Product \& Brand Management, Vol. 16 No. 2, pp. 152-3. 
Buil, I., Martínez, E., \& De Chernatony, L. (2010). Medición del valor de marca desde un enfoque formativo. Cuadernos de gestión, 10(1), 167-196.

Burkhalter, B.B. (1996). How Can Institutions of Higher Education Achieve Quality Within the New Economy? Total Quality Management, Vol. 7, No. 4, pp. 153-160.

Butin, D.W. (2010). Service-learning in theory and practice: The future of community engagement in higher education. Palgrave Macmillan.

Camacho, J. (2008): El valor de la marca: Brand Equity. Datos, Diagnóstico y Tendencias. Nielsen. http://mx.nielsen.com/press.

Camisón, C. (1998). Calidad y normalización en la pequeña y mediana empresa, Libro Blanco de la Pequeña y Mediana Empresa de la Comunidad Valenciana. Valencia: CEPYMEV/CIERVAL.

Camisón, C. (1999). Total Quality Management and Cultural Change: A Model of Organizational Development, International Journal of Technology Management. Vol. 16, No. 4-5-6, pp. 479-493.

Carmines, E.G., \& Zeller, R.A. (1979). Reliability and validity assessment, Vol. 17. Sage publications.

Caruana, A., Ramaseshan, B., \& Ewing, M.T. (1998). Do universities that are more market orientated perform better? International Journal of Public Sector Management, Vol. 11, No. 1, pp. 55-70.

Cervera, A., Schlesinger, W., Mesta, M.A., \& Sánchez, R. (2012). Medición de la imagen de la universidad y sus efectos sobre la identificación y lealtad del egresado: una aproximación desde el modelo de Beerli y Díaz (2003). Revista Española de Investigación en Marketing ESIC, No. 16(2), pp. 7-29.

Chen, L.H. (2008). Internationalization or international marketing? Two frameworks for understanding international students ${ }^{\text {ee }}$ choice of Canadian universities, Journal of Marketing for Higher Education, Vol. 18 No. 1, pp. 1-33.

Clotfelter, C.T. (2014). Buying the best: Cost escalation in elite higher education. Princeton University Press.

Coleman, J.S., Campbell, E., Hobson, C.J., Mcpartland, J., Mood, A.M., Weinfeld, F.dD., \& York, R. (1966). Equality of educational opportunity. Washington, DC: U.S. Government Printing Office.

Conway, T., Mackay, S., \& Yorke, D. (1994). Strategic planning in higher education: Who are the customer? The International Journal of Educational Management. Bradford, Vol. 8, No. 6, pp. 29-37.

Crosby, P. (1979). Quality is Free. The Art ofMaking Quality Certain. Nueva York: McGraw-Hill.

Cubillo, J.M., Sánchez, J., \& Cerviño, J. (2006). International students' decision-making process. International Journal of Educational Management, 20(2), 101-115.

Currás, R. (2007). Comunicación de la responsabilidad social corporativa: imagen e identificación con la empresa como antecedentes del comportamiento del consumidor.

Deem, R., \& Brehony, K.J. (2005). Management as ideology: The case of 'new managerialism' in higher education. Oxford review of education, Vol. 31(2), pp. 217-235.

Delgado, E., \& Munuera, J.L. (2002). Medición del capital de marca con indicadores formativos. Investigación y Markerting, Vol. 759, pp.16-20.

Deming, W.E. (1989). Calidad, Productividad y Competitividad. La salida de la crisis. Madrid: Díaz de Santos.

Denegri, M., Etchebarne, M.S., Geldres, V., Cabezas, D., \& Herrera, V. (2009). Personalidad de marca de las carreras de ciencias empresariales: un análisis corporativo entre universidad pública y privada. 
Douglas, J., Mcclelland, R., \& Davies, J. (2008). The development of a conceptual model of student satisfaction with their experience in higher education. Quality Assurance in Education, Vol. 16(1), pp. 19-35.

Duesterhaus, A.P., \& Duesterhaus, M. (2014). Attributes of successful university brands in the U.S.A. Journal of Brand Strategy, 3(2), 169-183.

Edo, M.T.G., Puig, V.R., \& Zornoza, C.C. (1999). Hacia modelos de calidad de servicio orientados al cliente en las universidades públicas: el caso de la Universitat Jaume I. Investigaciones Europeas de Dirección y Economía de la Empresa, Vol. 5(2), pp. 69-92.

Faircloth, J.b., Capella, L.m., \& Alford, B.1. (2001). The effect of brand attitude and brand image on brand equity. Journal of Marketing Theory and Practice, pp. 61-75.

Farquhar, P.H. (1989). Managing brand equity. Marketing research, Vol. 1(3).

Feigenbaum, A.V. (1983). Total Quality Control. New York: McGraw-Hill.

Ferrer, C.S. (2005). La percepción del cliente de los elementos determinantes de la calidad del servicio universitario: características del servicio y habilidades profesionales. Papeles del psicólogo, No. (90), 1.

Flavián, C., \& Lozano, F.J. (2002). Análisis de la relación entre la actitud y el comportamiento orientado al mercado en la universidad pública española. XIV Encuentro de Profesores Universitarios de Marketing, 531-546.

Fornell, C., \& Larcker, D.F. (1981). Structural equation models with unobservable variables and measurement error: Algebra and statistics. Journal of marketing research, pp. 382-388.

Furey, S., Springer, P., \& Parsons, C. (2009). University Brand Promises, Presentation at Academy Of Marketing 2009 Conference.

Gómez, M.L.N., \& Gutiérrez, O.D.M. (2001). Un análisis microeconómico de la demanda de educación superior en España. Estudios de economía aplicada, Vol. 19, pp. 69-86.

Gómez, D.F.H., \& Medina, R.Z. (2010). Diagnóstico de la imagen de marca de las instituciones universitarias en España.

Grijalba, M.A., \& López, M.R (2007). Evolución de la educación universitaria en España: diferentes perspectivas y principales tendencias (1991-2005). Revista de educación, No. 344, pp. 219-221.

Hair, J.F., Black, W.C., Babin, B.J., Anderson, R.E., \& Tatham, R.L. (2005). Multivariate data analysis. Upper Saddle River, NJ: Prentice Hall. Vol. 7.

Hamann, D., Williams, R., \& Omar, M. (2007). Branding Strategy and Consumer High-Technology Product, The Journal of Product \& Brand Management, 16, (Winter/Spring), Vol. 2, pp. 98-111.

Harvey, L., \& Green, D. (1993). Defining quality, Assessment and Evaluation in Higher Education, Vol. 18(1), pp. 9-34.

Helgesen, O. (2008). Marketing for higher education: A relationship marketing approach. Journal of Marketing for Higher Education, Vol. 18(1), pp. 50-78.

Hemsley-Brown, J., \& Goonawardana, S. (2007). Brand harmonization on the international higher education. Journal of Business Research, Vol. 60 No. 9, pp. 942-948.

Hemsley-Brown, J., \& Oplatka, I. (2006). Universities in a competitive global marketplace. A systematic review of the literature on higher education Marketing. International Journal of Public Sector Management, Vol. 19, No. 4, pp. 316-338.

Hennig-Thurau, T., Langer, M.F., \& Hansen, U. (2001). Modeling and Managing Student Loyalty An Approach Based on the Concept of Relationship Quality. Journal of service research, Vol. 3(4), pp. 331-344.

Hunger, J.D., Sánchez, T.L.W.M.A., Mejía, G.V.D.L.H., \& Hunger, J.D. (2007). Administracion Estrategica Y Politica de Negocios 10ed. Pearson Educación. 
Instituto Valenciano de Estadística (2008). La contribución socioeconómica de las universidades valencianas. Valencia: IVE.

Ishikawa, K. (1994). Introducción al Control de Calidad. Madrid: Díaz de Santos.

Jevons, C. (2006). Universities: a prime example of branding gone wrong. Journal of Product \& Brand Management, Vol. 15 No. 7, pp. 466-447.

Juran, J.M., \& Gryna, E.M. (1993). Manual de Control de Calidad. Madrid: McGraw-Hill Interamericana de España.

Keller, K.L. (1993). Conceptualizing, measuring, and managing customer-based Brand equity, Journal of Marketing, Vol. 57, January, pp. 1-22.

Küster, I. (2012). El Docente Universitario desde una perspectiva de mercado: Influencia en el rendimiento del estudiante. Alicante: 3 ciencias, pp. 1-118.

Küster, I., Vila, N., \& Aldás, J. (2011). Brand Equity Innovation: el uso de las nuevas tecnologías en el sector del vino para el incremento del valor de marca. Distribución y Consumo, No. 116, pp. 67 (pp. 1-15 en www.mercasa.es).

Lerman, D., \& Garbarino, E. (2002). Recall and Recog- nition of Brand Names: A Comparison of Word and Nonword Name Types, Psychology \& Marketing, Vol. 19 (7/8), pp. 621.

Lovelock, C.H. (1983). Classifying services to gain strategic marketing insights. The Journal of Marketing, pp. 9-20.

Lovelock, C.H., \& Dorfsman, I. (1997). Mercadotecnia de servicios. México: Prentice Hall, pp. $245-253$.

Lozier, G.G., \& Teeter, D.J. (1996). Quality improvement pursuits in American higher education. Total Quality Management, Vol. 7(2), pp. 189-202.

Mai, L.W. (2005). A comparative study between UK and US: The student satisfaction in higher education and its influential factors. Journal of Marketing Management, 21(7-8), 859-878.

Mcalexander, J.H., Koenig, H.F., \& Schouten, J.W. (2006). Building relationships of brand community in higher education: a strategic framework for university advancement. International Journal of Educational Advancement, 6(2), 107-118.

Miquel, S., Bigne, E., Sánchez, J., Moliner, M.A., \& Vallet, T.M. (1995). El marketing de los servicios públicos administrativos. Incomunicación presentada al IX Congreso Nacional-V Congreso Hispano-Francés, AEDEM, Toledo, pp. 2285-2299.

Mircea, M., \& Andreescu, A.I. (2011). Using cloud computing in higher education: A strategy to improve agility in the current financial crisis. Communications of the IBIMA, 2011, pp. 1-15.

Mourad, M., Ennew, C., \& Kortam, W. (2011). Brand equity in higher education. Marketing Intelligence \& Planning, Vol. 29(4), pp. 403-420.

Nguyen, N., \& Leblanc, G. (2001). Image and reputation of higher education institutions in students' retention decisions. International Journal of Educational Management, Vol. 15(6), pp. 303-311.

Nunnally, J.C., \& Bernstein, I. H. (1994). Psychometric theory. Third Edition. McGraw- Hill. New York.

Olins, W. (2008). Wally Olins: the brand handbook. Thames \& Hudson.

Owlia, M.S., \& Aspinwall, E.M. (1996). Quality in Higher Education-a Survey, Total Quality Management, Vol. 7, No. 4, pp. 161-171.

Paswan, A.k., \& Ganesh, G. (2009). Higher education institutions: Satisfaction and loyalty among international students. Journal of Marketing for Higher Education, Vol. 19(1), pp. 65-84.

Peltier, J.W., Schibrowsky, J.a., \& Drago, W. (2007). The interdependence of the factors influencing the perceived quality of the online learning experience: A causal model. Journal of Marketing Education, 29(2), pp. 140-153. 
Perez, F., \& Serrano, L. (1998). Capital Humano, Crecimiento Económico y Desarrollo Regional en España (1964-1997). Valencia: Fundación Bancaja.

Pinar, M., Trapp, P., Girard, T., \& Boyt, A.T. (2014). University Brand Equity: An Empirical Investigation of its Dimensions, Staying Current with Media \& Millennials, Vol. 50.

Rauschnabel, P.A., Krey, N., Babin, B.J., \& Ivens, B.S. (2016). Brand management in higher education: the university brand personality scale. Journal of Business Research, 69(8), 3077-3086.

Rojas, J.I., Vasquez, A.Z., Kara, A.L.I., \& Cerdá, A. (2009). Determinants of student loyalty in higher education: A tested relationship approach in Latin America. Latin American Business Review, Vol. 10(1), pp. 21-39.

Steiger, J.h. (1990). Structural model evaluation and modification: An interval estimation approach. Multivariate behavioral research, 25(2), 173-180.

Smith. B.E. (2003). An investigation of the market orientation of colleges and universities in the council of Christian colleges and universities. Doctoral dissertation, Capella University. (UMI Microform Service No 3129598).

Stripling, J. (2010). Brand new dilemma, available at: http://insidehighered.com/ News/2010/10/ 19/Branding (Accessed June 27, 2013).

Sultan, P., \& Yin Wong, H. (2012). Service quality in a higher education context: an integrated model. Asia Pacific Journal of Marketing and Logistics, Vol. 24(5), pp. 755-784.

Sung, M., \& Yang, S.u. (2008). Toward the model of university image: The influence of brand personality, external prestige, and reputation. Journal of Public Relations Research, Vol. 20(4), pp. 357-376.

Svenson, G., \& Wood, G. (2007). Are university students really customers? When illusion may lead to delusion for all. International Journal of Educational Management, Vol. 21, No. 1, pp. 17-28.

Thorsteinsson, G., Page, T., \& Niculescu, A. (2010). Using Virtual Reality for Developing Design Communication, Studies in Informatics and Control, Vol. 19 (1), pp. 93-106.

Toma, J.d., Dubrow, G., \& Hartley, M. (2005). The uses of institutional culture: Strengthening identification and building brand equity in higher education. Wiley Periodicals, Inc.

Waeraas, A., \& Solbakk, M. (2009): Defining the essence of a university: lessons from higher education branding, Higher Education, Vol. 57 No. 4, pp. 449-462.

Waterman, A. S. (2014). Service-learning: Applications from the research. Routledge.

Webb, D., \& Jagun, A. (1997). Customer care, customer satisfaction, value, loyalty and complaining behavior: validation in a UK university setting. Journal of consumer satisfaction, dissatisfaction and complaining behavior, 10, 139-151.

Whisman, R. (2009). Internal branding: a university's most valuable intangible asset, Journal of Product \& Brand Management, Vol. 18 No. 5, pp. 367-370.

Williams, R.1., \& Omar, M. (2009). Renaming Service Organizations for Growth, Presentation at Academy of Marketing 5th International Colloquium: Brand, Identity and Corporate Reputation, University of Cambridge, U.K.

Williams, R.1., Williams, H.a., \& Omar, M. (2013). The Marketing Impact of the Principles of Renaming Within a Higher Education Service Organization, American Marketing Association.

Yoo, B., \& Donthu, N. (2001). Developing and validating a multidimensional consumer-based brand equity scale. Journal of business research, Vol. 52(1), pp. 1-14.

Zeithaml, V.a., Bitner, M.j., De Lara Choy, M.i.p., Hirschfeld, A.1., \& Becerril, S.p. (2002). Marketing de Servicios: Un enfoque de integración del cliente a la empresa. México: McGraw-Hill. 
Zlotkowski, E. (1998). Successful Service-Learning Programs. New Models of Excellence in Higher Education. Anker Publishing Company, Inc. 176 Ballville Road, PO Box 249, Bolton, MA 01740-0249. 


\section{ANEXOS. ESCALAS DE MEDIDA DEL MODELO}

\section{F1. Notoriedad de marca universitaria}

NOT1. Grado de escucha positiva a otras personas sobre la propia institución educativa superior.

NOT2. Opinión sobre la propia institución educativa superior.

NOT3. Nivel de recomendación a realizar estudios de grado en la propia institución educativa superior.

NOT4. Nivel de recomendación a realizar estudios de postgrado en la propia institución educativa superior.

NOT5. Nivel de recomendación a realizar estudios de Doctorado en la propia institución educativa superior.

\section{F2. Imagen de marca universitaria}

IMAG1. Opinión sobre las personas que trabajan y estudian en la institución.

IMAG2. Razones para trabajar en la propia institución en lugar de otra similar.

IMAG3. Grado de confianza que transmite la propia institución educativa.

IMAG4. Nivel de prestaciones que ofrece la propia institución educativa.

IMAG5. Años de historia de la propia institución educativa superior.

IMAG6. Grado de satisfacción de necesidades del servicio la propia institución educativa superior.

IMAG7. Grado de eficacia y eficiencia del servicio la propia institución educativa superior.

IMAG8. Percepción de precios ofertados la propia institución educativa.

IMAG9. Nivel de facilidades y mantenimiento del servicio de la propia institución educativa superior.

IMAG10. Percepción sobre el deseo de otras personas por trabajar en la propia institución educativa superior.

IMAG11. Grado de crecimiento profesional junto a la propia institución.

F3. Calidad percibida de marca universitaria

CAL1. Opinión sobre el valor calidad-precio de la propia institución educativa.

CAL2. Opinión sobre el valor horas de trabajo-salario de la propia institución.

CAL3. Grado de satisfacción completa de necesidades del servicio la propia institución educativa superior.

CAL4. Opinión sobre el grado de relevancia de la marca educativa.

CAL5. Opinión sobre el grado de innovación del personal docente e investigador de la propia institución educativa superior.

CAL6. Opinión sobre el grado de interés hacia el alumnado del personal docente e investigador de la propia institución educativa superior.

CAL7. Opinión sobre el grado de interés hacia sus compañeros de trabajo del personal docente e investigador de la propia institución educativa superior.

CAL8. Opinión sobre el grado de admiración y respeto a la propia institución educativa superior del personal docente e investigador.

CAL9. Opinión sobre el grado de innovación del personal de administración y servi- 
cios de la propia institución educativa superior.

CAL10. Opinión sobre el grado de interés hacia el alumnado del personal de administración y servicios de la propia institución educativa superior.

CAL11. Opinión sobre el grado de interés hacia sus compañeros de trabajo del personal de administración y servicios de la propia institución educativa.

CAL12. Opinión sobre el grado de admiración y respeto a la propia institución educativa superior del personal de administración y servicios.

CAL13. Grado de recomendación de la propia institución a otras personas.

CAL14. Opinión sobre las ventajas que ofrece trabajar en la propia institución.

CAL15. Grado de emoción por trabajar en la propia institución educativa.

CAL16. Grado de diversión por trabajar en la propia institución educativa.

CAL17. Grado de calidez al trabajar en la propia institución educativa superior.

CAL18. Grado de seguridad laboral de la propia institución educativa superior.

CAL19. Grado de estabilidad laboral de la propia institución educativa superior.

CAL20. Grado de auto-respeto al trabajar en la propia institución superior.

CAL21. Grado de aprobación social al trabajar en la propia institución superior.

\section{F4. Lealtad de marca universitaria}

LEAL1. Grado de orgullo de que los demás sepan que trabaja en la propia institución educativa superior.

LEAL2. Grado de satisfacción en cuanto a expectativas profesionales.

LEAL3. Nivel de satisfacción en cuanto a sentimientos que transmite la propia marca educativa superior.

LEAL4. Percepción adecuada para considerar especial a la propia institución educativa superior.

LEAL5. Nivel de identificación con los valores de la propia institución educativa superior.

LEAL6. Nivel de identificación con los valores de los compañeros de trabajo de la propia institución educativa superior.

LEAL7. Grado de pertenencia a la propia institución educativa superior.

LEAL8. Grado de conexión con los compañeros de trabajo de la propia institución educativa superior.

LEAL9. Grado de interés por hablar de la propia institución educativa superior a otras personas.

LEAL10. Grado de interés en aprender más sobre la propia institución educativa superior.

LEAL11. Grado de interés por seguir las noticias de la propia institución educativa superior.

F5. Capital de marca universitario

CM1. Grado de conocimiento de la propia institución educativa superior.

CM2. Opinión sobre el valor que suministra la institución educativa en base al dinero que se paga.

CM3. Opinión positiva sobre la propia institución educativa superior.

CM4. Grado de lealtad a nivel profesional a la propia institución educativa. 